\title{
Aspectos epidemiológicos da hepatite B no município de Teresina
}

\author{
Epidemiological aspects of Hepatitis B in the city of Teresina
}

\author{
Aspectos epidemiológicos de la Hepatitis B en la ciudad de Teresina
}

Camila Brígida de Abreu Evangelista ${ }^{1}$, Gabriela Dantas Carvalho ${ }^{1 *}$, Kamila Cristiane de Oliveira Silva ${ }^{1}$, Tiago da Rocha Oliveira ${ }^{2}$, Neusa Barros Dantas Neta ${ }^{1}$, Augusto César Evelin Rodrigues ${ }^{1}$, Valéria Alves da Rocha¹, Marcélia Gomes Silva1, Érica Melo Lima1, Rayssa Maria de Araujo Carvalho'.

\section{RESUMO}

Objetivo: Traçar o perfil epidemiológico da infecção por hepatite B na cidade de Teresina-PI. Métodos: Tratase de um estudo documental, quantitativo e descritivo, realizado no período de junho a outubro de 2020, através dos casos de hepatite B notificados no banco de dados do SINAN, na versão SINAN NET, disponibilizados pelo Departamento de Informática do Sistema Único de Saúde (DATASUS), no período de 2014 a 2018. Os dados foram organizados em planilha digital Microsoft Office Excel 2019 e apresentados em tabelas, ordenadas por caracteres quantitativos, adotando como critério de distribuição a amostra e porcentagem, com o auxílio dos programas computacionais TabWin versão 4.1.5. Resultados: Foram contabilizados 32 casos, com maior incidência para os anos de 2014 e 2017, ambos reagentes para o marcador sorológico HBsAg, tendo predomínio para o gênero feminino, idade entre 20 e 39 anos e manifestado na forma crônica, sendo a atividade sexual a principal via de transmissão. Conclusão: Teresina tem alta endemicidade, com prevalência para a idade, meio de contaminação e diagnóstico clínico manifestado.

Palavras-chave: Hepatite B, Epidemiologia, Infecção.

\begin{abstract}
Objective: To trace the epidemiological profile of hepatitis B infection in the city of Teresina-PI. Methods: This is a documentary, quantitative and descriptive study, carried out from June to October 2020, using hepatitis B cases reported in the SINAN database, in the SINAN NET version, made available by the Department of Informatics of the Unified Health System. Health (DATASUS), from 2014 to 2018. The data were organized in a Microsoft Office Excel 2019 digital spreadsheet and presented in tables, ordered by quantitative characters, adopting the sample and percentage as distribution criteria, with the aid of the TabWin computer programs. version 4.1.5. Results: 32 cases were accounted for, with a higher incidence for the years 2014 and 2017, both of which are reagents for the HBsAg serological marker, with a predominance of females, aged between 20 and 39 years and manifested in the chronic form, with sexual activity being the main route transmission. Conclusion: Teresina has high endemicity, with prevalence for age, means of contamination and manifest clinical diagnosis.
\end{abstract}

Keywords: Hepatitis B, Epidemiology, Infection.

RESUMEN

Objetivo: Trazar el perfil epidemiológico de la infección por hepatitis B en la ciudad de Teresina-PI. Métodos: Se trata de un estudio documental, cuantitativo y descriptivo, realizado de junio a octubre de 2020, utilizando los casos de hepatitis B reportados en la base de datos SINAN, en la versión SINAN NET, puesta a disposición por el Departamento de Informática del Sistema Único de Salud. Salud (DATASUS), de 2014 a 2018. Los datos se organizaron en una hoja de cálculo digital Microsoft Office Excel 2019 y se presentaron en tablas, ordenadas por caracteres cuantitativos, utilizando la muestra y el porcentaje como criterio de distribución, con la ayuda de los programas informáticos TabWin. versión 4.1.5. Resultados: Se contabilizaron 32 casos, con mayor incidencia para los años 2014 y 2017, ambos reactivos para el marcador serológico HBsAg, con predominio para el sexo femenino, con edades entre 20 y 39 años y manifestados en la forma crónica, con siendo la actividad sexual la principal vía de transmisión. Conclusión: Teresina tiene alta endemicidad, con prevalencia por edad, medio de contaminación y diagnóstico clínico manifiesto.

Palabras clave: Hepatitis B, Epidemiología, Infección.

${ }^{1}$ Centro Universitário UNIFACID, Teresina - PI. *E-mail: ftgabrieladantas@hotmail.com

2 Centro Universitário INTA (UNINTA), Sobral - CE. 


\section{INTRODUÇÃO}

O termo hepatite viral refere-se aos vírus hepatotrópicos primários, caracterizados por apresentar uma estrutura envelopada, que infecta apenas os seres humanos. Atualmente, são conhecidos cinco vírus responsáveis pelas diferentes hepatites humanas: os vírus das hepatites A (HAV), B (HBV), C (HCV), D (HDV) e E (HEV), que pertencem, respectivamente, às famílias Picornaviridae, Hepadnaviridae, Flaviviridae, Deltaviridae e Hepeviridae (BRASIL, 2014). Estes vírus são capazes de desencadear doenças hepáticas de espectro diversificado, podendo evoluir de forma aguda (até mesmo fulminante) ou crônica, com manifestação assintomática ou sintomática, dependendo do agente viral envolvido e dos fatores imunogenéticos do paciente (FERREIRA AR, et al., 2014).

A transmissão do vírus da HBV ocorre de forma vertical e depende da interação hospedeiro-vírus, podendo ocorrer através das lesões na pele e na mucosa, que se dá pelo contato direto com o material biológico infectado, preferencialmente sangue ou sêmen, tendo como as principais vias de transmissão: mães infectadas para os neonatos, parceiros sexuais e por mecanismos invasivos, tais como uso de injeções, transfusões de sangue ou diálise (HATZAKIS A, et al., 2013).

O período de incubação é de 30 a 180 dias, com média de 60 a 90 dias, acometendo, preferencialmente, indivíduos na faixa etária de 20 a 40 anos (DUTRA RL e HAAS P, 1999), aos quais podem manifestar sinais e sintomas de forma insidiosos e variáveis, entre os mais frequentes sintomas estão o cansaço, tontura, enjoo e/ou vômitos, ou em casos mais graves apresentarem artralgias e exantemas, perda de apetite, dispepsia, dor abdominal, mialgia generalizada, mal-estar e fraqueza, podendo aparecer ou não a pele e olhos amarelados (LOPES TGSL, et al., 2011).

No Brasil, a taxa de mortalidade por hepatite B é de 0,6 por 100000 habitantes, sendo as regiões do Nordeste e Sudeste classificadas como áreas de endemicidade intermediária, seguidos do Espírito Santo e Santa Catarina, com alta endemicidade. O Ministério da Saúde estima que $15 \%$ da população brasileira já entrou em contato com HBV e que 1\% apresenta formas crônicas (BRASIL, 2016). A hepatite B representa um desafio à saúde pública mundial, estimando-se, aproximadamente, 350 milhões de indivíduos portadores crônicos desse vírus ao redor do mundo e um milhão de mortes por ano vítimas da doença, ou seja, 5\% da população do planeta, são portadores dessa virose (OSTI C e MACHADO JM, 2010).

Segundo a Organização Mundial de Saúde (OMS) apesar da imunoprofilaxia eficaz e do tratamento antiHBV, a infecção crônica por HBV ainda é uma grande ameaça em várias partes do mundo, necessitando do cuidado integral e da articulação dos serviços e ações de saúde para ampliar o cuidado, prevenção e o acompanhamento do usuário infectado (WHO, 2018).

Nesse contexto, a pesquisa objetiva traçar o perfil epidemiológico da infecção por hepatite $B$ na cidade de Teresina-PI.

\section{MÉTODOS}

Trata-se de um estudo documental, epidemiológico, observacional e descritivo, realizado no período de junho a outubro de 2020, com caráter quantitativo. A população deste estudo foi composta pelos casos confirmados de hepatite B notificados no banco de dado do Sistema de Informação de Agravos e Notificação do Ministério da Saúde (SINAN/PI), na versão SINAN NET, disponibilizados pelo Departamento de Informática do Sistema Único de Saúde (DATASUS), no período de 2014 a 2018.

A partir dos dados levantados foram estratificadas as seguintes variáveis: incidência anual, gênero, faixa etária, fonte de infecção, manifestação clínica da doença e presença do marcador sorológico HbsAg, mantendo as correlações para o marcador AntiHBclgM e excluindo AntiHCV.

Foi adotado como critério de inclusão os casos de hepatite $B$ confirmados laboratorialmente. Como critério de exclusão, os casos de hepatite $B$ associados a outras hepatites e pertencentes a outros municípios do estado do Piauí.

Os dados foram organizados em planilha digital Microsoft Office Excel 2019 e apresentados em tabelas, ordenadas por caracteres quantitativos, adotando como critério de distribuição a amostra e porcentagem, com o auxílio dos programas computacionais TabWin versão 4.1.5. 


\section{RESULTADOS E DISCUSSÃO}

Conforme dados do Sistema de Informações SINAN/PI no período entre 2014 a 2018 a distribuição dos casos notificados e confirmados de hepatite B em Teresina contabilizou 32 casos, mostrando uma maior incidência para o ano de 2014 (31,25\%) e 2017 (31,25\%), com 10 casos cada. Quanto ao gênero predominante, destaca-se o feminino $(n=19,59,37 \%)$, tendo para o mesmo uma maior incidência em 2014 $(n=9,28,12 \%)$ e 2017 para o masculino $(n=8,25 \%)$ (Tabela 1).

Tabela 1 - Casos confirmados de hepatite B segundo o sexo.

\begin{tabular}{cccc}
\hline Ano & Masculino & Feminino & Total \\
\hline $\mathbf{2 0 1 4}$ & 1 & 9 & 10 \\
$\mathbf{2 0 1 5}$ & 1 & 4 & 5 \\
$\mathbf{2 0 1 6}$ & 3 & 2 & 5 \\
$\mathbf{2 0 1 7}$ & 8 & 2 & 10 \\
$\mathbf{2 0 1 8}$ & 0 & 2 & 2 \\
\hline Total & 13 & 19 & 32 \\
\hline
\end{tabular}

Fonte: Evangelista CBA, et al., 2021; dados extraídos de Sistema de Informação de Agravos e Notificação do Ministério da Saúde (SINAN), 2020.

Segundo os dados levantados, em Teresina há o predomínio de infecção do gênero feminino, dado semelhante foi encontrado no estudo de Nicolau S, et al. (2017), ao qual de forma semelhante fez o levantamento epidemiológico dos casos de hepatite B com bases nos dados ofertados pelo SINAN entre os anos de 2011 e 2015 no estado de Pernambuco, apresentando uma incidência de 59,8\% dos casos para o gênero feminino contra $40,2 \%$ para o masculino, para a mesma faixa etária.

Estudo realizado por Araújo TME, et al. (2012) investigaram a vulnerabilidade dos adolescentes entre 14 a 19 anos à hepatite $\mathrm{B}$ no município de Teresina-PI, mostrando 109 (55,6\%) casos afetados para o gênero feminino em comparação aos $87(44,4 \%)$ casos para o gênero masculino, onde a sua maioria apresentava faixa etária entre 14 a 16 anos $(n=113 ; 57,7 \%)$, sendo a maioria solteira $(n=182 ; 92,9 \%)$, com baixa escolaridade (100\%), não vacinadas $(n=120,61,2 \%)$ e naturais do município de Teresina ( $n=168 ; 85,7 \%)$.

Concomitante aos dados apresentados, Magalhães LBM (2013) em sua tese de doutorado, investigou o percentual de infecção pela HBV no gênero feminino em Teresina e a cobertura vacinal, mostrando que dentre as infectadas $63,4 \%$ referiram não ter um companheiro fixo, evidenciando um baixo nível de instrução (67,16\%), onde $11,7 \%$ eram analfabetas e que afirmaram não terem sido vacinadas, justificando não terem se apresentado na data marcada para a realização da vacinação.

Diante do exposto, o perfil dos infectados no município de Teresina sugere ser um perfil característico local, uma vez que ao analisar os dados apresentados na literatura, com abordagem analítica dos perfis dos infectados pela hepatite $B$ em outras regiões do Brasil, mostra um predomínio ao gênero masculino (BRASIL 2016a; BRASIL 2017).

O perfil apresentado pelo município de Teresina sugere estar diretamente relacionado ao perfil socioeconômico da população afetada, uma vez que, dentre os estudos realizados, observa-se que o maior percentual dos casos de hepatite B está para a população com baixa escolaridade, ausência de um parceiro fixo e que não participaram da vacinação, mostrando que o grau de instrução é um fator importante para o desenvolvimento de políticas sanitárias, visto que a intervenção primária dada pela educação em saúde à população uma vez bem desenvolvida consegue prevenir a disseminação de infecções e, consequentemente, conter danos e agravos.

Segundo o Boletim Epidemiológico das Hepatites Virais (BRASIL, 2019) do total de casos de hepatite B notificados de 1999 a 2015, 106.371 (54,1\%) são entre homens, percentual este que condiz com os resultados apresentados por Santos ACS e Morais MTM (2017) que justificam que a hepatite B tem uma maior incidência para o gênero masculino por vários fatores, dentre estes figuram as questões biológicas e sociais que lhes propiciam uma atividade sexual com maior número de parceiras(os), estilo de vida relacionado ao maior consumo de bebidas alcoólicas, hábito de fumar e ausência de preservativo, além do fato da maioria da população masculina não procurar serviços de saúde (CARVALHO JR, et al., 2014; VIEIRA GD, et al., 2015). 
Segundo Sá Ml, et al. (2015) o cuidado, por questões socioculturais, está mais voltado à mulher e por se acreditarem superiores e resistentes, os homens são mais vulneráveis a diversas doenças. Logo, acredita-se na possibilidade da subnotificação frente ao descaso do gênero masculino diante do processo de notificação no município de Teresina.

Outro importante fator que deve ser considerado dentre os dados apresentados é a precisão quanto aos dados obtidos no sistema de vigilância. Farias N, et al. (2011) descrevem que ao apurar dados epidemiológicos deve-se considerar a frágil estrutura do sistema de vigilância epidemiológica local. O sistema de vigilância das hepatites virais constitui um sistema passivo e convive com as subnotificações, de tal forma que os casos registrados não são representativos da realidade da população geral, portanto, não devem ser usados para inferências populacionais, refletindo a atividade e a adesão das notificações pelos serviços de saúde, o que poderia justificar o alto percentual de mulheres com hepatite B em Teresina, ou ainda, pode-se sugerir a relação entre o gênero feminino e o perfil socioeconômico como fator determinante para a população afetada.

No entanto, dentre os dados levantados quanto aos infectados com hepatite B no município de Teresina, observa-se a alta parcela dos indivíduos não são vacinados, ou que não receberam as 3 doses de vacina, em especial, o gênero feminino. Segundo Trivello R, et al. (1995) as medidas de segurança, tais como sexo com preservativo, o cuidado no contato com conteúdo biológico ou instrumento perfurocortante infectados são eficazes, mas não excluem a vacinação, uma vez que a vacinação contra hepatite B é altamente imunogênica e protetora contra a infecção e induz uma resposta adequada em, aproximadamente, $90 \%$ dos adultos e $95 \%$ das crianças.

Visando confirmar os números de casos confirmados para os referidos anos, foi analisado o percentual do marcador HBsAg. Segundo Heiat M, et al. (2014) o diagnóstico da hepatite B é dado através da detecção dos marcadores presentes no sangue, soro, plasma ou fluido oral da pessoa infectada, por meio de imunoensaios, e/ou na detecção do ácido nucleico viral, empregando técnicas de biologia molecular, sendo confirmado quando o indivíduo apresenta um ou mais dos marcadores reagentes: HBsAg reagente (incluindo teste rápido reagente), Anti-HBc IgM reagente ou HBV-DNA detectável (BRASIL, 2019).

$\mathrm{O}$ estudo detectou que todos os notificados para hepatite $\mathrm{B}$ foram reagentes para o marcador $\mathrm{HBsAg}$ $(\mathrm{N}=32,100 \%)$, seguidos de 3 casos negativos, não se enquadrando nos infectados pelo HBV e 7 suspeitos, porém, sem análise sanguínea (Tabela 2).

Tabela 2 - Casos de hepatite B por Marcador Sorológico HBsAg, notificados no Município de Teresina.

\begin{tabular}{cccc}
\hline Ano & Reagente & Não reagente & Não realizado \\
\hline $\mathbf{2 0 1 4}$ & 10 & 1 & 1 \\
$\mathbf{2 0 1 5}$ & 5 & 0 & 3 \\
$\mathbf{2 0 1 6}$ & 5 & 1 & 0 \\
$\mathbf{2 0 1 7}$ & 10 & 1 & 2 \\
$\mathbf{2 0 1 8}$ & 2 & 0 & 1 \\
\hline Total & 32 & 3 & 7
\end{tabular}

Fonte: Evangelista CBA, et al., 2021; dados extraídos de Sistema de Informação de Agravos e Notificação do Ministério da Saúde (SINAN), 2020.

A infecção pelo HBV é considerada alta onde a prevalência do HBsAg é superior a $7 \%$, ou onde $60 \%$ ou mais da população têm evidência sorológica de infecção prévia (CHÁVEZ JH, et al., 2003). No Brasil as taxas de detecção das regiões vêm apresentando tendência de aumento desde o início da notificação compulsória, sendo a região Sul com a maior taxa dentre as regiões do país, enquanto que o Nordeste apresenta a menor taxa de deteç̧ão em todo o período (BRASIL, 2016).

A prevalência de hepatite $B$, determinada para presença do $\mathrm{HBsAg}$, é classificada como: áreas de baixa endemicidade (<2\%), intermediária baixa (2-4\%), intermediária alta (5-7\%) e alta ( $\geq 8 \%)$ (OTT et al., 2012). A prevalência do HBsAg nas capitais da região Nordeste do Brasil, baseado em inquérito nacional é 0,5 (BRASIL, 2010). O município de Teresina apresenta taxa de endemicidade de $8,6 \%$, sendo, neste contexto, classificada com alta endemicidade, tendo prevalência mínima de 6,25\% nos casos anuais notificados. 
Segundo a OMS, o Brasil é classificado como de baixa endemicidade, com prevalência de $0,07 \%$ (WHO, 2018), no entanto, quando se analisa em aspectos regionais, $45 \%$ dos detectados apresentam positivo para HBsAg igual ou superior a 8\% (CDC, 2019).

Na distribuição por ano e faixa etária mostra-se que, ao longo do período estudado, o grupo mais atingido foram os indivíduos entre 20 e 39 anos ( $N=20,62,5 \%)$, seguidos da faixa etária entre 40 e 59 anos ( $n=7$, 21,87\%) (Tabela 3). Dados semelhantes são observados no Boletim Epidemiológico de Hepatite Virais 2019 (BRASIL, 2019), na qual cita uma maior incidência a contaminação do HBV para indivíduos entre 20 a 29 anos (CARLO FS, et al., 2008; JUSTINO EMG, et al., 2014).

Vieira GD, et al. (2015) justificam que por considerar o período da população sexualmente ativa evidenciado por taxas elevadas em idade reprodutiva, o que facilitaria o contágio, em especial, aqueles cuja relação não se faz com proteção e/ou entre múltiplos parceiros (COFFIN C, et al., 2012), esses dados coadunam com informações de outros estudos em que predominam a HB começando aos 16 anos de idade (COSTA LG, et al., 2012).

Araújo TME e Silva NC (2014) investigaram o perfil dos profissionais da enfermagem submetidos a acidentes de trabalho perfurocortantes e as medidas preventivas para a contaminação de hepatite B, mostrando prevalência para o gênero feminino e com idade entre 41 a 50 anos, onde apenas $51,1 \%$ encontravam-se vacinadas com as 3 doses, seguido das doses incompletas (32,5\%), ou que não teriam sido vacinadas $(7,3 \%)$ ou mesmo não lembram $(9,1)$, identificando o possível perfil de indivíduos acometidos com hepatite B com faixa etária entre 40 a 59 anos.

Tabela 3 - Casos confirmados de hepatite B segundo a idade.

\begin{tabular}{|c|c|c|c|c|c|c|c|}
\hline \multirow{2}{*}{ Ano } & \multicolumn{7}{|c|}{ Variáveis } \\
\hline & $20-39$ & $40-59$ & $60-64$ & $65-69$ & $70-79$ & $>80$ anos & Total \\
\hline 2014 & 7 & 1 & 0 & 1 & 0 & 1 & 10 \\
\hline 2015 & 3 & 1 & 0 & 1 & 0 & 0 & 5 \\
\hline 2016 & 4 & 1 & 0 & 0 & 0 & 0 & 5 \\
\hline 2017 & 6 & 2 & 1 & 0 & 1 & 0 & 10 \\
\hline 2018 & 0 & 2 & 0 & 0 & 0 & 0 & 2 \\
\hline Total & 20 & 7 & 1 & 2 & 1 & 1 & 32 \\
\hline
\end{tabular}

Fonte: Evangelista CBA, et al., 2021; dados extraídos de Sistema de Informação de Agravos e Notificação do Ministério da Saúde (SINAN), 2020.

Esta pesquisa mostra que a via sexual foi à segunda fonte de infecção mais prevalente $(n=7,21,87 \%)$. É importante observar que o grande número de casos encontrados com relação ao mecanismo de transmissão está ignorado ou não respondido $(n=20,62,5 \%)$. Situação semelhante em um estudo realizado por Cerqueira EM, et al. (2010) em Feira de Santana - BA e Nicolau S, et al., (2017) em Recife - PE, aos quais foram encontradas notificações incompletas (Tabela 4).

Tabela 4 - Casos confirmados de hepatite B segundo a causa de infecção.

\begin{tabular}{cccccccc}
\hline Ano & Sem resposta & Sexual & Transfusional & Vertical & $\begin{array}{c}\text { Tratamento } \\
\text { cirúrgico }\end{array}$ & $\begin{array}{c}\text { Alimento/ } \\
\text { água }\end{array}$ & Total \\
\hline $\mathbf{2 0 1 4}$ & 5 & 5 & 1 & 0 & 0 & 0 & 10 \\
$\mathbf{2 0 1 5}$ & 5 & 0 & 0 & 0 & 0 & 0 & 5 \\
$\mathbf{2 0 1 6}$ & 3 & 0 & 0 & 0 & 1 & 0 & 5 \\
$\mathbf{2 0 1 7}$ & 5 & 2 & 0 & 2 & 0 & 1 & 10 \\
$\mathbf{2 0 1 8}$ & 2 & 0 & 0 & 0 & 0 & 0 & 2 \\
\hline Total & 20 & 7 & 1 & 2 & 1 & 1 & 32 \\
\hline
\end{tabular}

Fonte: Evangelista CBA, et al., 2021; dados extraídos de Sistema de Informação de Agravos e Notificação do Ministério da Saúde (SINAN), 2020.

Nota-se uma limitada confiabilidade para os dados apurados dada a subnotificação, uma vez que no ato do preenchimento as informações são ignoradas e/ou deixadas em branco. A baixa adesão ao preenchimento da via de transmissão é uma forma de subnotificação que deve ser combatida e leva a crer que os percentuais podem ser maiores no município de Teresina. 
A dificuldade na notificação dos casos é referida no Boletim Epidemiológico de Hepatites Virais de 2019 (BRASIL, 2019), onde mostram que nos anos de 2014, 2015 e 2017, o campo das referidas informações teve aproximadamente $25 \%$ do preenchimento total, o que mostra uma baixa adesão, dificultando a precisão epidemiológica local (NICOLAU S, et al., 2017).

Diante disso, surge o alerta para capacitação de profissionais, com a finalidade de conhecerem a importância dos registros e implementarem medidas de controle sobre a doença.

Em relação ao diagnóstico clínico, 65,6\% $(n=21)$ apresenta ser portador da hepatite crônica, tendo maior incidência no ano de 2014, com 7 casos, seguido de 18,7\% $(n=6)$ para a forma aguda e 15,6\% ( $n=5)$ com diagnóstico inconclusivo, sendo estes, possivelmente, diagnosticados como hepatite B após alguns meses da conduta tomada com novos estudos sorológicos (Tabela 5).

Tabela 5 - Casos confirmados de hepatite B segundo o diagnóstico clínico.

\begin{tabular}{ccccc}
\hline Ano & Hepatite aguda & Hepatite crônica & Inconclusivo & Total \\
\hline $\mathbf{2 0 1 4}$ & 0 & 7 & 3 & 10 \\
$\mathbf{2 0 1 5}$ & 0 & 4 & 1 & 5 \\
$\mathbf{2 0 1 6}$ & 2 & 3 & 0 & 5 \\
$\mathbf{2 0 1 7}$ & 4 & 5 & 1 & 10 \\
$\mathbf{2 0 1 8}$ & 0 & 2 & 0 & 2 \\
\hline Total & 6 & 21 & 5 & 32 \\
\hline
\end{tabular}

Fonte: Evangelista CBA, et al., 2021; dados extraídos de Sistema de Informação de Agravos e Notificação do Ministério da Saúde (SINAN), 2020.

O HBV pode manifestar distúrbios hepáticos agudos e crônicos, onde após a incubação viral, os indivíduos infectados desencadeiam um quadro de hepatite aguda, onde, em torno de, 90 a $95 \%$ desses casos evolui para a cura e 5\% a $10 \mathrm{HBV}$ crônico (FERREIRA AR, et al., 2014).

O diagnóstico de ambas as formas clínicas dar-se por meio de técnicas sorológicas, avaliadas como fundamentais não só pelo diagnóstico, mas também por mostram-se úteis no seguimento da infecção viral, na avaliação do estado clínico do paciente e na monitorização da terapêutica específica (HOOFNAGLE JH e DI BISCEGLIE AM, 1991).

$\mathrm{Na}$ fase aguda ocorre intensa replicação viral, podendo manifestar-se tanto nas formas sintomáticas e ictéricas da doença, quanto nas formas anictéricas e oligossintomáticas. O período de incubação varia de 2 a 6 meses, onde após cerca de 6 semanas da contaminação, o AgHbs já se encontra presente no soro, podendo permanecer positivo por até 180 dias nos casos agudos, quando então desaparece e é substituído pelo anticorpo anti-Hbs após algumas semanas ou meses depois. Em média, de 5\% a 10\% dos pacientes que persistem com o AgHbs no soro além de 6 meses são avaliados como portadores crônicos do vírus (HOOFNAGLE JH e DI BISCEGLIE AM, 1991).

O diagnóstico para hepatite $B$ dificulta a tomada de decisão dada a alta incidência de indivíduos assintomáticos, podendo assim mascarar o percentual dos diagnósticos para as formas agudas e crônicas. Moschetta F e Peres MA (2007) ao analisar os prováveis infectados com hepatite B constataram que $91,2 \%$ eram portadores assintomáticos para vírus B. De forma semelhante, Viana DR, et al. (2017) ao analisarem o perfil clínico-epidemiológico de idosos na região Nordeste, verificou um maior número de casos para assintomáticos e de fase crônica. Contudo, quando investigada a prevalência quanto as formas aguda ou crônica manifestada no Brasil, observa-se predominância na forma crônica (BRASIL, 2018; PIMENTA MMKG, et al., 2019).

O desenvolvimento da hepatite $B$ crônica é definida sorologicamente como persistência do antígeno de superfície do HBV-AgHbs e está atrelada à baixa imunidade (GOMES SRL, 2017), ou ainda ao difícil diagnóstico apresentado pelos casos assintomáticos que permitem a ação do vírus sobre o organismo (PIMENTA MMKG, et al., 2019). Segundo Alvariz RC, (2006), a fase crônica da hepatite B é determinada pela replicação viral e a resposta imune produzida de maneira inadequada, ou ainda, condições que podem acelerar a progressão da infecção, tais como o gênero feminino e a gravidez (PIMENTA MMKG, et al., 2019). 
Observa-se que na longa evolução dos pacientes com hepatite B crônica, a presença de duas fases bem distintas: a primeira corresponde a períodos mais precoces da doença, nesta, o HBV demonstra intensa replicação, identificada pela presença do antígeno "e" (AgHbe), do AgHbs no soro, do DNA viral (DNA-HBV); além da biópsia hepática alterada com atividade inflamatória portal e periportal que pode variar de leve a intensa, que depende da manifestação imunológica do paciente (REALDI G, et al., 1984).

Parte dos pacientes portadores da hepatite $B$ não apresentam doença hepática, no entanto, a outra parcela desenvolve sinais de atividade inflamatória no fígado, que se manifestam de variada intensidade por anos, podendo desencadear um quadro de cirrose hepática e/ou hepatocarcinoma nas fases mais avançadas da doença (DI MARCO V, et al., 1999). Quanto aos sintomas apresentados não foram configurados no estudo, uma vez que o levantamento epidemiológico realizado pelo banco de dados do SINAN não apresenta tais informações.

\section{CONCLUSÃO}

As notificações epidemiológicas realizadas pelo sistema de informação do SINAN apesar de ser um instrumento norteador sobre as políticas públicas, apresenta limitação quanto a veracidade dos dados apresentados, muitos destes subnotificados, o que dificulta a percepção sobre as condições de saúde local. Contudo, por meio deste pode-se perceber que Teresina tem alta endemicidade, entrando em consenso, em sua maioria, com dados apresentados na literatura, onde mostram prevalência para a idade, meio de contaminação e diagnóstico clínico manifestado. Considerando que a hepatite B é uma doença de notificação compulsória, faz desta necessária a seriedade de medidas quanto a sua notificação, garantindo a ação da vigilância epidemiológica, como forma de prevenir a doença e controlar seu processo de contágio.

\section{REFERÊNCIAS}

1. ALVARIZ RC. Hepatite Crônica pelo Vírus B (HBV). Rev HUPE, 2006; 5(1): 16-34.

2. ARAÚJO TME, et al. Análise da vulnerabilidade dos adolescentes à hepatite $B$ em Teresina/PI. Rev. Eletr. Enf. [Internet], 2012; 14(4): 873-82.

3. ARAÚJO TME, SILVA NC. Acidentes perfurocortantes e medidas preventivas para hepatite B adotadas por profissionais de Enfermagem nos serviços de urgência e emergência de Teresina, Piauí. Rev. bras. Saúde ocup., 2014; 39(130): 175-183.

4. CARVALHO JR, et al. Método Para Estimação de Prevalência de Hepatites B e C Crônicas e Cirrose Hepática - Brasil, 2008. Epidemiologia E Serviço Saúde, 2014; 23(4): 1-9.

5. CARLO FS, et al. Perfil do Portador de Hepatite B do Município de Maringá. Rev Saúde Pesquisa, $2008 ; 1: 241-46$.

6. BRASIL. Secretaria de Vigilancia em saúde. Ministério da Saúde. Departamento de DST, AIDS e Hepatites Virais. Estudo de prevalência de base populacional das infecções pelos vírus das hepatites A, B e C nas capitais do Brasil. Universidade de Pernambuco, Brasília, DF: [s.n.], 2010, 211p. Disponível em: http://periodicos.ses.sp.bvs.br/pdf/bepa/v4n44/v4n44a05.pdf Acesso: 17 mar. 2021.

7. BRASIL. Ministério da Saúde (BR). Secretaria de Vigilância em Saúde. Guia de vigilância em saúde. Brasília: Ministério da Saúde; 2014. Hepatites virais; p. 311-24. Disponível em: https://bvsms.saude.gov.br/bvs/publicacoes/guia_vigilancia_saude_3ed.pdf Acesso em: 17 mar. 2021.

8. BRASIL. Ministério da Saúde. Secretaria de Vigilância em Saúde - Departamento de Vigilância, Prevenção e Controle das DST, Aids e Hepatites Virais (DDAHV). Boletim Epidemiológico: Hepatites Virais. Brasília: Ministério da Saúde, 2016. Disponível em: http://www.aids.gov.br/pt-br/pub/2016/boletim-epidemiologico-de-hepatites-virais-2016. Acessado em: 13 dez. 2020.

9. BRASIL. Secretaria Estadual de Saúde. Secretaria Executiva de Vigilância em Saúde. Diretoria Geral de Promoção, Monitoramento e Avaliação da Vigilância em Saúde. Perfil Socioeconômico, Demográfico e Epidemiológico. 1. ed. Pernambuco, Recife: Secretaria de Saúde do Estado de Pernambuco, 2016a. Disponível em: http://portal.saude.pe.gov.br/secretaria-executiva/secretaria-executiva-de-vigilancia-em-saude. Acesso em: 17 mar. 2021.

10. BRASIL. Ministério da Saúde. Secretaria de Vigilância em Saúde. Boletim Epidemiológico: Hepatites Virais. Ministério da Saúde. Secretaria de Vigilância em Saúde. Brasília: Ministério da Saúde, v. 48, p.24, 2017. Disponível em: http://www.aids.gov.br/pt-br/pub/2019/boletim-epidemiologico-de-hepatites-virais-2017. Acesso em: 17 mar. 2021.

11. BRASIL. Ministério da Saúde. Protocolo de Recomendação. Protocolo Clínico e Diretrizes Terapêuticas para Profilaxia Pós Exposição de Risco à Infecção pelo HIV, IST e Hepatites Virais. Secretaria de Ciência, Tecnologia e Insumos Estratégicos. Brasília, 2018; Disponível em: http://www.aids.gov.br/pt-br/pub/2015/protocolo-clinico-e-diretrizesterapeuticas-para-profilaxia-pos-exposicao-pep-de-risco. Acessado em: 9 nov. 2020.

12. BRASIL. Ministério da Saúde. Boletim Epidemiológico: Hepatites Virais. Brasília: Ministério da Saúde, 2019. Disponível em: http://www.aids.gov.br/pt-br/pub/2019/boletim-epidemiologico-de-hepatites-virais-2019. Acessado em: 22 nov. 2020. 
13. CENTERS FOR DISEASE CONTROL AND PREVENTION (CDC). Pinkbook. Hepatitis B. Epidemiology of Vaccine Preventable Diseases, 2019. Disponível em: https://www.cdc.gov/vaccines/pubs/pinkbook/hepb.html. Acessado em: 12 dez. 2020.

14. CERQUEIRA EM, et al. Ocorrência de hepatites virais na microrregião de Feira de Santana, Bahia. Rev B.S. Publica Miolo, 2010; 34(4): 980-89.

15. CHÁVEZ JH, et al. Panorama da Hepatite B no Brasil e no Estado de Santa Catarina. Rev Panamericana Saúde Pública, 2003; 14(2): 91-6.

16. COSTA LG, et al. Detecção de Infecção pelo vírus da hepatite $B$ nos municípios brasileiros segundo cobertura dos serviços de hemoterapia, no período de 2011 a 2008. Epidemiol. Serv. Saúde 2012; 21(4): 617-26.

17. DI MARCO V, et al. The long-term course of chronic hepatitis B. Hepatology, 1999; 30: 257-264.

18. DUTRA RL, HAAS P. Hepatite B no Estado de Santa Catarina. News Lab, 1999; 7(35): 102-104.

19. FARIAS N, et al. Casos de Hepatite B e $C$ notificados em adolescentes ao Sistema Nacional de Informação de Agravos do estado de São Paulo, de 2007 a 2010. BEPA, Bol. epidemiol. Paul, 2011; 8(93): 4-11.

20. FERREIRA AR, et al. Hepatites Virais A, B e C em crianças e adolescentes. Rev Méd Minas Gerais, 2014; 24(2): 4660.

21. GOMES SRL. Caracterização dos Genótipos e Subgenótipos do Vírus da Saber Científico. Hepatite B em Portadores Crônicos do Estado de Rondônia, 2017; 8(1): 41-49.

22. HATZAKIS A, et al. The State of Hepatitis B and C in the Mediterranean and Balkan Countries: Report from a Summit Conference The State of Hepatitis B and C in the Mediterranean and Balkan Countries: Journal of Viral Hepatitis, 2013; 20(2): 1-20.

23. HEIAT, M, et al. Classical and Modern Approaches Used for Viral Hepatitis Diagnosis. Hepat Mon, 2014; 14(4): 1763217651.

24. HOOFNAGLE JH, DI BISCEGLIE AM. Serologic diagnosis of acute and chronic viral hepatitis. Seminars of Liver Disease, 1991; 11: 73-83.

25. JUSTINO EMG, et al. Perfil de Portadores de Hepatite B em um Serviço de Referência: Estudo Retrospectivo. Rev Bras Promoç Saúde, 2014; 27(1): 53-61.

26. LOPES TGSL, et al. Aspectos gerais da hepatite B. R. Ci. med. biol., 2011; 10(3): 337-44.

27. MOSCHETTA F, PERES MA. Perfil Epidemiológico dos Portadores de Hepatite B no Município de Chapecó-SC no Período de 1996 a 2006. Florianópolis: Diretoria de Vigilância Epidemiológica, 2007.

28. MAGALHÃES RLB. Infecção pelo vírus da hepatite B, HIV e cobertura vacinal em profissionais do sexo feminino em Teresina - PI. Universidade de São Paulo, Ribeirão Preto, 2013; 387 p.

29. NICOLAU S, et al. Perfil Epidemiológico da Hepatite B em uma Regional de Saúde em Recife. Rev. Saúde Col. UEFS, $2017 ; 7(3): 30-35$

30. OSTI C, MACHADO JM. Vírus da hepatite B: avaliação da resposta sorológica à vacina em funcionários de limpeza de hospital-escola. Ciência \& Saúde Coletiva, 2010; 15(1): 1343-1348.

31. OTT JJ, et al. Global Epidemiology of Hepatitis B Virus Infection: New Estimates of Age-Specific HBsAg Seroprevalence and Endemicity. Vaccine, 2012; 30(12): 2212-2219.

32. PIMENTA MMKG, et al. Hepatite B: Distribuição Epidemiológica no Estado de Rondônia no Período de 2008 a 2017. Saber Científico, 2019; 8: 41-49.

33. REALDI G, et al. Seroconversion from hepatitis B e antigen to anti-Hbe in chronic hepatitis B virus infection. Gastroenterology, 1984; 86: 230-235.

34. SÁ MI et al. Infecções sexualmente transmissíveis e factores de risco nas adolescentes e jovens: Dados de um Centro de Atendimento a Jovens. Nascer e Crescer, 2015; 24(2): 64-69.

35. SANTOS ACS, MORAIS MTM. Perfil Epidemiológico e Sociodemográfico dos Portadores de Hepatite B de um Município do Sudoeste Baiano. Rev. Saúde.Com, 2018; 14(1): 1073-1080.

36. TRIVELLO R, et al. Persistence of anti-AgHBs antibodies in health care personnel to recombinant DNA HB booster vaccine. Vaccine, 1995; 13(2): 139-141.

37. VIANA DR, et al. Hepatite B e C: Diagnóstico e tratamento. Revista de Patologia do Tocantins, 2017; 4(3): 73-79.

38. VIEIRA GD, et al. Hepatitis B in Rondônia (Western Amazon Region, Brazil): Descriptive Analysis and Spatial Distribution. Arq. Gastroenterol, 2015; 52: 18-21.

39. WHO. World Health Organization. Hepatitis B. Geneva: World Health Organization, 2018. Disponível em: https://apps.who.int/iris/handle/10665/67746. Acessado em: 11 dez. 2020. 